\title{
Endoscopic Removal of a Fecalith or Fecomucolith in a Rectal Stump or Pouch
}

\author{
Evie Yeap ${ }^{1,2}$, Ratheesraj Ratinam ${ }^{1}$, Asiri Arachchi ${ }^{1,2}$, James Lim ${ }^{1}$, Yeng Kwang Tay ${ }^{1}$, Zeev Duieb ${ }^{1}$ \\ ${ }^{1}$ Department of Colorectal Surgery, Monash Health, Dandenong Hospital, Dandenong, VIC; ${ }^{2}$ Department of Surgery, School of Clinical \\ Sciences at Monash Health, Monash University, Clayton, VIC, Australia
}

Fecaliths or fecomucoliths can form in rectal stumps after a Hartmann procedure or in a coloneovaginal conduit. They can cause significant distress to the patient by causing symptoms such as discharge and odor. We describe a novel and effective method of endoscopic obliteration and removal where other techniques such as removal during examination under anesthesia or serial enemas have failed. By using a combination of hydrodissection with a saline injector gun and biopsy forceps and a polypectomy snare to break down the fecomucolith or fecalith, this troublesome problem can be resolved endoscopically. Successful removal of the fecalith/fecomucolith and resolution of the symptoms for the patients were achieved. No complications are reported. We describe an effective and novel method for endoscopic removal of fecaliths and fecomucoliths.

Keywords: Gastrointestinal endoscopy; Fecalith; Colonoscopy

\section{INTRODUCTION}

Fecaliths and fecomucoliths can form in rectal stumps post-Hartmann's procedure and other procedures such as coloneovaginoplasty. They are formed from retained stool and/or mucus in the bowel lumen which hardens over time. They can cause significant symptoms and when large and present for some time, they can be very difficult to treat. We describe a method that can be used when other methods such as enemas and removal of fecomucolith under anesthetic fails. An effective endoscopic method of obliteration and removal in this setting has not been published. However, a similar technique has previously been described for the removal of a fecalith at the appendiceal orifice via colonoscopy $[1,2]$. Our endoscopic method is useful where other techniques such as removal with a pair of forceps under anesthesia or serial enemas have failed. Informed consent for publication has

Received: Mar 13, 2021 - Revised: Jun 17, 2021 - Accepted: Jul 11, 2021 Correspondence to: Evie Yeap, MBBS (Hons), PGradDipSurgAnat Department of Colorectal Surgery, Monash Health, Dandenong Hospital, 135 David Street, Dandenong, VIC 3175, Australia

Tel: +61-3-95548657, Fax: +61-3-95541177

E-mail: evie.yeap@monashhealth.org

ORCID: https://orcid.org/0000-0002-4989-0011

(c) 2021 The Korean Society of Coloproctology

This is an open-access article distributed under the terms of the Creative Commons Attribution NonCommercial License (https://creativecommons.org/licenses/by-nc/4.0) which permits unrestricted noncommercial use, distribution, and reproduction in any medium, provided the original work is properly cited. been obtained from the patient and this article has been written in line with institutional ethical guidelines.

\section{TECHNIQUE}

We report the use of a multi-method approach to this problem, which is particularly useful where other methods such as self-administering enemas or an examination under anesthetic and removal of fecalith fails due to stenosis at the anastomosis.

A 57-year-old male to female sex reassignment patient presented with offensive smelling discharge from a neovagina. This had caused significant distress and affected her personal and sexual relationships. The patient had previously undergone surgery 18 years prior where the penis skin was inverted to create a vaginal cavity. She then subsequently had a coloneovaginoplasty to increase the vaginal depth (Fig. 1). The patient was not performing the required daily neovaginal dilatation to prevent stenosis. She had delayed seeking medical treatment due to concern about stigma by wider community and from the medical profession despite the considerable effect of the odor and discharge on her selfesteem and relationships.

The cause for the presenting complaint was a large fecomucolith that had formed above the coloneovaginal anastomosis which was stenotic (Fig. 1). Other more traditional methods were initially used. The fecomucolith was unable to be grasped with Rampleys forceps under anesthesia and this only caused advancement of the 

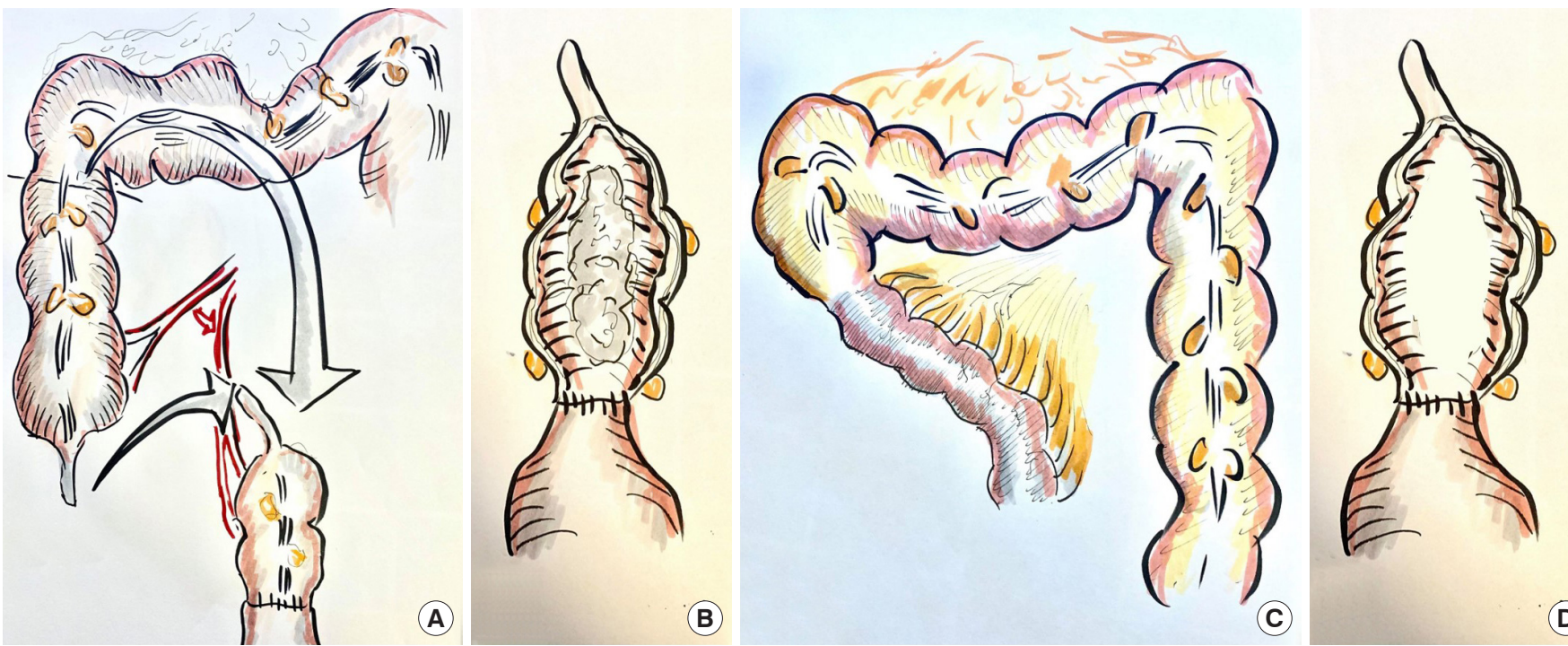

Fig. 1. Anatomy of colovaginoplasty pouch. (A) Ascending colon mobilized for colovaginoplasty. (B) Colovaginoplasty with anastomosis between ascending colon and neovagina and fecomucolith trapped above stenotic anastomosis. (C) Ileocolic anastomosis after the segment of ascending colon is used to form colovaginoplasty. (D) Appearance of the coloneovaginal conduit with fecomucolith removed.
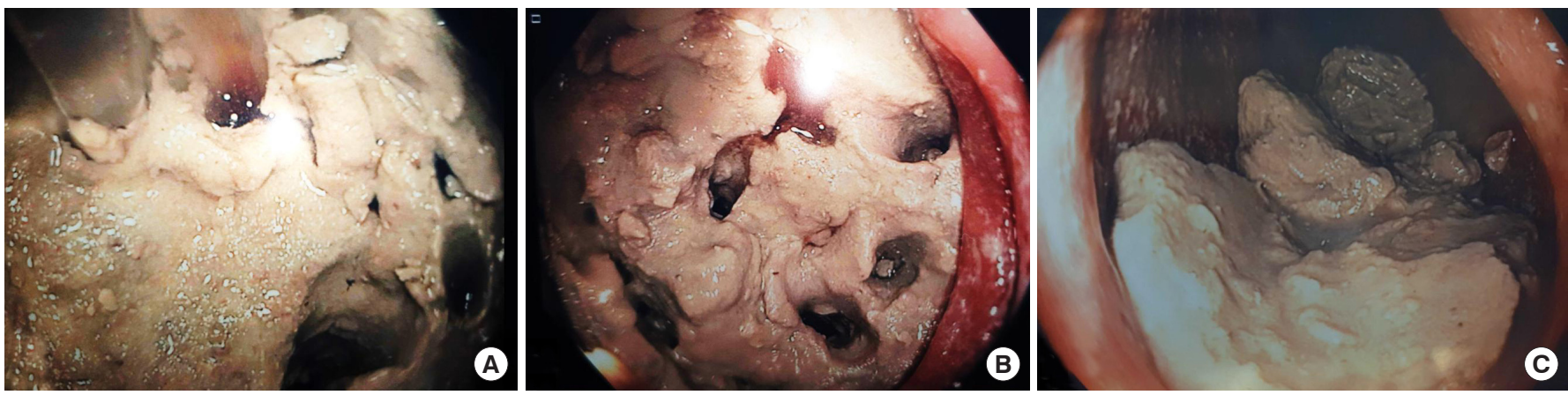

Fig. 2. Endoscopic images demonstrating removal of fecomucolith. (A) Hydrodissection of fecomucolith using a saline injector gun. (B) Multiple holes within the fecomucolith created with the saline injector. (C) After saline injection and using forceps to break up the fecomucolith, it can be removed in pieces.

fecomucolith to an inaccessible point. Further, stenosis of the anastomosis made passing instruments difficult. Enemas into the neovaginal cavity were also ineffective in removing the fecomucolith.

Therefore, a novel endoscopic method was developed which can be used where other methods such as removal under anesthetic and enemas have failed as in this case. The same technique has also been used by our group for an elderly patient with a fecalith within a rectal stump following a Hartmann procedure.

A colonoscope was passed to the point of the fecomucolith. Several techniques were used to break down and remove the fecomucolith. Firstly, the Interject Clear 23 gage injection needle catheter and saline injector gun (Boston Scientific, Marlborough, MA, USA) are used to hydrodissect holes into the fecomucolith (Fig. 2). This is done by injection in the same area with a high-pressure saline push. Multiple holes can be formed in the fecalith with this method. Then, large biopsy forceps are used to break down the fecalith by inserting the jaws closed into the holes and opening the jaws to fracture it. A large polypectomy snare (Captivator Extra Large Rounded Stiff braided 33-mm Polypectomy Snare; Boston Scientific) is used to break the fecalith into smaller pieces and shave softer segments of the fecalith off (Fig. 2). Finally, a retrieval net is used to retrieve the smaller pieces. Finally, a flushing pump and suction action are used to suction larger fragments onto the end of the scope to facilitate removal.

\section{DISCUSSION}

The fecalith was able to be successfully removed without any complications in this patient and the presenting symptoms re- 


\section{Coloproctology Evie Yeap, et al.}

solved. The same result was achieved in the elderly patient who presented with a fecalith within the rectal stump after a Hartmann procedure. The risk of perforation of the colonic conduit is very low with this technique.

We recommend performing this procedure under general anesthetic in the lithotomy position. The surgeon should allow at least 2 to 3 hours for the case and it is not suitable for a routine endoscopy list. Our tips for this procedure include keeping the pouch well-inflated with air and irrigation fluid and pressing the fecalith into the apex of the conduit to prevent it from slipping away.

This technique is an effective and novel method for endoscopic removal of a fecalith or fecomucolith when removal under anesthetic and enemas have failed. Using a stepwise approach with multiple methods of dissection, this troublesome problem can be solved with readily available endoscopic devices.

\section{CONFLICT OF INTEREST}

No potential conflict of interest relevant to this article was reported.

\section{REFERENCES}

1. Hsu CW. Colonoscopic removal of a giant appendiceal faecolith without appendectomy for acute appendicitis - a video vignette. Colorectal Dis 2018;20:1056.

2. Kurt M, Posul E, Can G, Yilmaz B, Korkmaz U. Removal of appendiceal fecalith with colonoscopy. Gazi Med J 2016;27:156-7. 